\title{
Robust high-gain DNN observer for non- linear stochastic continuous time systems
}

\author{
D.A. Murano, A.S. Poznyak, Lennart Ljung
}

Division of Automatic Control

E-mail: , apoznyak@ctrl.cinvestav.mx, ljung@isy.liu.se

25th June 2007

Report no.: LiTH-ISY-R-2803

Accepted for publication in IEEE Conference on Decison and Control, 2001

Address:

Department of Electrical Engineering

Linköpings universitet

SE-581 83 Linköping, Sweden

WWW: http://www.control.isy.liu.se

AUTOMATIC CONTROL

REGLERTEKNIK

LINKÖPINGS UNIVERSITET

Technical reports from the Automatic Control group in Linköping are available from http://www. control.isy.liu.se/publications. 


\begin{abstract}
A class of nonlinear stochastic processes satysfying a "Lipschitz-type strip condition" and supplied by a linear output equation, is considered. Robust asymptotic (high-gain) state estimation for nonlinear stochastic processes via differential neural networks is discussed. A new type learning law for the weight dynamics is suggested. By a stochastic Lyapunov-like analysis (with Ito formula implementation), the stability conditions for the state estimation error as well as for the neural nertwork weights are established. The upper bound for this error is derived. The numerical example, dealing with "module"-type nonlinearities, illustrates the effectiveness of the suggested approach.
\end{abstract}

Keywords: observers, neural networks 


\title{
Robust High-Gain DNN Observer for Nonlinear Stochastic Continuous Time Systems
}

\author{
Daishi A. Murano ${ }^{\dagger}$, Alex S. Poznyak ${ }^{\dagger}$ and Lennart Ljung ${ }^{\ddagger}$ \\ † CINVESTAV-IPN, Dept. of Automatic Control, AP 14 740, CP 07000, \\ Mexico D.F., Mexico, e-mail: apoznyak@ctrl.cinvestav.mx \\ $\ddagger$ Linköping University, Dept. of Electr. Eng.S-581 83, Linköping, Sweden \\ e-mail: ljung@isy.liu.se
}

\begin{abstract}
A class of nonlinear stochastic processes, satisfying a "Lipschitz-type strip condition" and supplied by a linear output equation, is considered. Robust asymptotic (high-gain) state estimation for nonlinear stochastic processes via differential neural networks is discussed. A new type learning law for the weight dynamics is suggested. By a stochastic Lyapunov-like analysis (with Itô formula implementation), the stability conditions for the state estimation error as well as for the neural network weights are established. The upper bound for this error is derived. The numerical example, dealing with "module"-type nonlinearities, illustrates the effectiveness of the suggested approach.
\end{abstract}

Keywords: Dynamic Neural Networks, Stochastic Processes, Nonlinear Observers.

\section{Introduction}

Due to many successful applications, the Neural Network technique seems to be a very effective for identification and controlling of a wide class of complex nonlinear systems especially in the absence of a complete model information ("grey-box" models) or, even, considering a controlled plant as "a black box" (Hunt et al., 1992 [6]). In general, the NN's can be qualified as static (feedforward) and as dynamic (recurrent or differential) nets. The most of recent publications (see, for example, Haykin, 1994 [7]; Agarwal, 1997 [1]; Parisini and Zoppoli, 1994. [15]) deals with Static NN, providing the appropriate approximation of a nonlinear operator functions in the right-hand side of dynamic model equations. In spite of successful implementations, they have several important disadvantages such as a slow learning rate (the weights updates do not utilize the information on the local NN structure) and a high sensitivity of the function approximation to the training data (they do not have memory, so their outputs are uniquely determined by the current inputs and weights). Dynamic Neural Networks can successfully overhead these draw- backs and demonstrate a workable behavior in the presence of essential unmodelled dynamics because their structure incorporate feedback. They have been introduced by Hopfield, 1984 [5] and then studied in (Sandberg, 1991 [23](the approximation aspects), Rivithakis and Christodoulou, 1994 [21] (the direct adaptive regulation via DNN), Poznyak et al., 1999 [19](the adaptive tracking using DNN)). Several advance results concerning DNN have been recently obtained in (Narendra and Li, 1998 [14](the identification and control), Lewis and Parisini, 1998 [11] (NN feedback control: the stability analysis) and in (Rivithakis and Christodoulou, $2000[22]$ ). All of these publications concern NN applications considered specifically only for the external environment of the deterministic nature. There are a few publications dealing with the identification or control of stochastic processes with unknown dynamics via NN (see Kosmatopulos and Christodoulou, 1994 [9] and Elanayar and Shin, 1994 ). In the recent paper of the authors (Poznyak and Ljung, 2001 [20]) the identification of continuous time stochastic system via DNN has been studied but under the assumption that all state measurements are available and are not disturbed by a noise component.

In this paper we spread the ideas of DNN approach to the class of continuous time stochastic processes (with incomplete dynamic model description) based only on the output measurement subjected to stochastic noise corruptions. Dynamic Neural Networks given by differential equations (Differential Neural Networks) are applied to obtain the on-line state estimates. In fact, a robust high-gain DNN observer is suggested. The main goal of this paper is to show that practically the same robust DNN observers, designed for the deterministic systems with bounded perturbations, is robust with respect to perturbations of a stochastic nature which are unbounded (with probability one). The stochastic calculus (such as Itô formula and the martingale technique together with the strong large number law implementation) are shown to be the effective instrument for the solution of this problem. 


\section{Uncertain Stochastic System}

Let $\left(\Omega, \mathcal{F},\left\{\mathcal{F}_{t}\right\}_{t \geq 0}, \mathbf{P}\right)$ be a given filtered probability space $\left((\Omega, \mathcal{F}, \mathbf{P})\right.$ is complete, the $\mathcal{F}_{0}$ contains all the $\mathbf{P}$ null sets in $\mathcal{F}$, the filtration $\left\{\mathcal{F}_{t}\right\}_{t>0}$ is right continuous: $\mathcal{F}_{t+}:=\bigcap_{s>t} \mathcal{F}_{s}=\mathcal{F}_{t}$ ). On this probability space define an $m$-dimensional standard Brownian motion, that is, $\left(\bar{W}_{t}, t \geq 0\right)$ (with $\left.\bar{W}_{0}=0\right)$ is an $\left\{\mathcal{F}_{t}\right\}_{t \geq 0}$-adapted $\mathbb{R}^{m_{-}}$ valued process such that

$$
\begin{gathered}
E\left\{\bar{W}_{t}-\bar{W}_{s} \mid \mathcal{F}_{s}\right\}=0 \quad \mathbf{P}-\text { a.s. } \\
\mathbf{P}\left\{\omega \in \Omega: W_{0}=0\right\}=1 \\
E\left\{\left[\bar{W}_{t}-\bar{W}_{s}\right]\left[\bar{W}_{t}-\bar{W}_{s}\right]^{\top} \mid \mathcal{F}_{s}\right\}=(t-s) I \quad \mathbf{P}-\text { a.s. }
\end{gathered}
$$

Consider the stochastic nonlinear controlled continuous-time system with the dynamics $x_{t}$ :

$$
\begin{gathered}
x_{t}=x_{0}+\int_{s=0}^{t} b\left(s, x_{s}, u_{s}\right) d t+\int_{s=0}^{t} \sigma^{x}\left(s, x_{s}, u_{s}\right) d \bar{W}_{s} \\
y_{t}=C x_{t}+\int_{s=0}^{t} \sigma^{y}\left(s, x_{s}, u_{s}\right) d \bar{W}_{s}
\end{gathered}
$$

or, in the abstract (symbolic) form,

$$
\left\{\begin{array}{l}
d x_{t}=b\left(t, x_{t}, u_{t}\right) d t+\sigma^{x}\left(t, x_{t}, u_{t}\right) d \bar{W}_{t} \\
x_{0}=x_{\text {given }}, \quad t \in[0, \infty) \\
d y_{t}=C d x_{t}+\sigma^{y}\left(t, x_{t}, u_{t}\right) d \bar{W}_{t}
\end{array}\right.
$$

The first integral in (1) is an stochastic ordinary integral and the second one is an Itô integral. In the above $x_{t} \in \mathbb{R}^{n}$ is system state at timet, $u_{t} \in U \subseteq \mathbb{R}^{p}$ is a control, $y_{t} \in \mathbb{R}^{k}$ is the output, $b:[0, \infty) \times \mathbb{R}^{n} \times U \rightarrow \mathbb{R}^{n}$, $\sigma^{x}:[0, \infty) \times \mathbb{R}^{n} \times U \rightarrow \mathbb{R}^{n \times m}, \sigma^{y}:[0, \infty) \times \mathbb{R}^{n} \times U \rightarrow$ $\mathbb{R}^{k \times m}$ and $C \in \mathbb{R}^{k \times n}$ is a constant matrix characterizing the state-output mapping. Below we will use the following notation: $\bar{W}_{t}^{x}:=\int_{s=0}^{t} \sigma^{x}\left(s, x_{s}, u_{s}\right) d \bar{W}_{s}, \bar{W}_{t}^{y}$ $:=\int_{s=0}^{t} \sigma^{y}\left(s, x_{s}, u_{s}\right) d \bar{W}_{s}$. It is assumed that

A1: $\left\{\mathcal{F}_{t}\right\}_{t \geq 0}$ is the natural filtration generated by $\left(\bar{W}_{t}, t \geq 0\right)$ and augmented by the $\mathbf{P}$-null sets from $\mathcal{F}$.

A2: $(U, d)$ is a separable metric space with a metric $d$.

Definition 1 The function $f:[0, \infty) \times \mathbb{R}^{n} \times U \rightarrow$ $\mathbb{R}^{n \times m}$ is said to be an $L_{\phi}^{A}\left(C^{2}\right)$-mapping (or, "stripcone" type) if it is Borel measurable and is $C^{2}$ in $x$ (almost everywhere) for any $t \in[0, \infty)$ and any $u \in U$ and also there exist a matrix $A \in \mathbb{R}^{n \times n}$, a constant $L$ and a modulus of continuity $\phi:[0, \infty) \rightarrow[0, \infty)$ such that for any $t \in[0, \infty)$ and for any $x, u, \hat{x}, \hat{u} \in \mathbb{R}^{n} \times U \times \mathbb{R}^{n} \times U$

$$
\begin{gathered}
\|f(t, x, u)-f(t, \hat{x}, \hat{u})-A(x-\hat{x})\| \leq \\
L\|x-\hat{x}\|+\phi(d(u, \hat{u})) \\
\left\|f_{x}(t, x, u)-f_{x}(t, \hat{x}, \hat{u})-A\right\| \leq L\|x-\hat{x}\|+\phi(d(u, \hat{u})) \\
\left\|f_{x x}(t, x, u)-f_{x x}(t, \hat{x}, \hat{u})\right\| \leq \phi(\|x-\hat{x}\|+d(u, \hat{u}))
\end{gathered}
$$

(here $f_{x}(\cdot, x, \cdot)$ and $f_{x x}(\cdot, x, \cdot)$ are the partial derivatives of the first and the second order).

A3: $b(t, x, u)$ is $L_{\phi}^{A}\left(C^{2}\right)$-mappings, $\sigma^{x}\left(t, x_{t}, u_{t}\right)$ and $\sigma^{y}\left(t, x_{t}, u_{t}\right)$ are $L_{\phi}^{0}\left(C^{2}\right)$-mappings (or, for simplicity, $\left.L_{\phi}\left(C^{2}\right)\right)$.

Definition 2 A stochastic control $u_{t}$ is called a feasible in the stochastic sense (or, s-feasible) for the system (2) if $u_{t} \in \mathcal{U}[0, \infty):=\left\{u:[0, \infty) \times \Omega \rightarrow U \mid u_{t}\right.$ is $\left\{\mathcal{B}_{t}\right\}_{t>0}$-adapted $\},\left\{\mathcal{B}_{t}\right\}_{t>0}$ is the filtration generated by $\left(\left(y_{\tau}, u_{\tau}: \tau \in[0, t]\right), t \geq 0\right)$ and augmented by the $\mathbf{P}$ - null sets from $\mathcal{F}$, and $x_{t}$ is the unique solution of (2) in the sense that for any $x_{t}$ and $\hat{x}_{t}$, satisfying (2), $\mathbf{P}\left\{\omega \in \Omega: x_{t}=\hat{x}_{t}\right\}=1$.

The set of all $s$-feasible controls is denoted by $\mathcal{U}_{\text {feas }}^{s}[0, \infty)$. The triple $\left(x_{t} ; y_{t} ; u_{t}\right)$, where $\left(x_{t}, y_{t}\right)$ is the solution of (2), corresponding to this $u_{t}$, is called an $s$-feasible triple. The assumptions A1-A3 guarantee that any $u_{t}$ from $\mathcal{U}[0, \infty)$ is $s$ feasible. It is assumed also that the past information about $\left(y_{\tau}, u_{\tau}: \tau \in[0, t]\right)$ is available for the controller: $u_{t}:=u\left(t, y_{\tau \in[0, t]}, u_{\tau \in[0, t)}\right)$ implying that $u_{t}$ becomes $\left\{\mathcal{F}_{t}\right\}_{t \geq 0}$-adapted too.

The only sources of uncertainty in this system description are the system random noise $\bar{W}_{t}$, the priori unknown $L_{\phi}^{A}\left(C^{2}\right)$-function $b(t, x, u)$ and $L_{\phi}\left(C^{2}\right)$ function $\sigma^{x, y}(t, x, u)$. The class $\Sigma_{u n}$ of uncertain stochastic systems (2) satisfying A1-A3 is considered below as the main object of the investigation.

\section{Differential Neural Networks Observer}

Consider the following DNN observer (Poznyak and $\mathrm{Yu}$, 2000 [16]) with a single hidden layer ${ }^{1}$ :

$$
\left\{\begin{array}{c}
d \hat{x}_{t} / d t=A \hat{x}_{t}+W_{1, t} \sigma\left(V_{1, t} \hat{x}_{t}\right)+W_{2, t} \varphi\left(V_{2, t} \hat{x}_{t}\right) \gamma\left(u_{t}\right) \\
+K\left(y_{t}-C \hat{x}_{t}\right)
\end{array}\right.
$$

where $\hat{x}_{t} \in \mathbb{R}^{n}$ is its state vector at time $t, \sigma: \mathbb{R}^{r} \rightarrow \mathbb{R}^{k}$ is the given $L_{\phi}\left(C^{2}\right)$-mapping, $\varphi: \mathbb{R}^{s} \rightarrow \mathbb{R}^{l}$ is the given $L_{\phi}\left(C^{2}\right)$-mapping, $V_{1, t} \in \mathbb{R}^{r \times n}, V_{2, t} \in \mathbb{R}^{s \times n}$ are a $\left\{\mathcal{B}_{t}\right\}_{t \geq 0}$-adapted adjustable internal hidden layer weight matrices, $W_{1, t} \in \mathbb{R}^{n \times k}, W_{2, t} \in \mathbb{R}^{n \times l}$ are a $\left\{\mathcal{B}_{t}\right\}_{t \geq 0^{-}}$adapted adjustable the output layer weight matrices, $\gamma: \mathbb{R}^{p} \rightarrow \mathbb{R}^{q}$ the given $L_{\phi}\left(C^{2}\right)$-mapping satisfying $\|\gamma(u)\| \leq \gamma_{0}+\gamma_{1}\|u\|$ for any $u \in U, A \in \mathbb{R}^{n \times n}$ is a constant matrix characterized by $L_{\phi}^{A}\left(C^{2}\right)$-mapping. The initial state vector $\hat{x}_{0}$ is supposed to be quadratically integrable $\left(E\left\{\left\|\hat{x}_{0}\right\|^{2}\right\}<\infty\right)$.

\footnotetext{
${ }^{1} \mathrm{~A}$ multi layer DNN can be represented in a single layer form by the corresponding matrix dimension extension.
} 
Remark 1 The mappings $\sigma$ and $\varphi$ are usually assumed to be of the main diagonal structure with the bounded sigmoidal elements $\sigma_{i i}$ and $\varphi_{i i}$, that is, $\sigma_{i i}:=$ $\frac{a_{i}}{1+e^{-b_{i}^{\top} \hat{x}}}-c_{i} \quad(i=1, \min \{r ; k\}), \bar{\sigma}:=\sup _{x \in \mathbb{R}^{n}}\|\sigma(x)\|$, $\bar{\varphi}:=\sup _{x \in \mathbb{R}^{n}}\|\varphi(x)\|$ which obviously belong to the class of $L_{\phi}\left(C^{2}\right)$-functions.

\section{The Learning Law for Identification Process}

The matrices collection $\left(A, V_{1, t}, V_{2, t}, W_{1, t}, W_{2, t}\right)$ should be selected to provide "a good enough" state estimation process for any possible uncertain stochastic system from $\Sigma_{u n}$.

Accept also some technical assumption which will be use in the main result formulation.

A4: the applied $s$-feasible control $u_{t} \in U$ is quadratically integrable (uniformly on $t$ ) as well the corresponding stochastic system dynamics $x_{t}$, that is, $\limsup _{t \rightarrow \infty}$ $\left[E\left\{\left\|u_{t}\right\|^{2}\right\}+E\left\{\left\|x_{t}\right\|^{2}\right\}\right]<\infty$. In fact, this assumption restricts the consideration by the class of BIBOstable systems (with bounded second moment of state vector).

A5: The pair $(A, C)$ is detectable.

A6: The gain matrix $K$ is selected such a way that the joint matrix $\bar{A}:=A-K C$ is stable and there exist positive definite matrices $\Lambda_{\sigma}, \Lambda_{3}, \Lambda_{1}, \Lambda_{2}, \Lambda_{f}, \Lambda_{y}, Q_{0}$ providing the existence of a positive solution to the following algebraic matrix Riccati equation

$$
\begin{aligned}
G & :=P \bar{A}+\bar{A}^{\top} P+P R P+Q=0 \\
R & :=2 \bar{W}_{1}+2 \bar{W}_{2}+\Lambda \Lambda_{y}^{-1} \Lambda^{\top}+\Lambda_{f}^{-1} \\
Q & :=\Lambda_{\sigma}+\delta \Lambda_{3}+\delta \Lambda_{y}+2 \delta \Lambda_{1}+Q_{0}
\end{aligned}
$$

has a positive solution $P=P^{\top}>0$. and $\bar{W}_{1}:=$ $W_{1,0} \Lambda_{1}^{-1} W_{1,0}^{\top}, \bar{W}_{2}:=W_{2,0} \Lambda_{2}^{-1} W_{2,0}^{\top}$.

A7. The large number law (Poznyak, 2000 [19]) is valid for the considered processes

$$
\left\{\begin{array}{c}
T^{-1} \int_{t=0}^{T}\left(\left\|x_{t}\right\|^{2}-E\left\|x_{t}\right\|^{2}\right) d t \stackrel{a . s .}{\rightarrow} 0 \\
T^{-1} \int_{t=0}^{T}\left(\left\|u_{t}\right\|^{2}-E\left\|u_{t}\right\|^{2}\right) d t \stackrel{a . s .}{\rightarrow} 0
\end{array}\right.
$$

The last assumptions is not so restrictive since the only decreasing of the autocorrelation functions of the considered processes is required to fulfill (5) that automatically holds for the accepted constructions (Poznyak et al, $2000[18])$.
Introduce the following notations:

$$
\begin{gathered}
\hat{\sigma}_{t}:=\sigma\left(V_{1, t} \hat{x}_{t}\right)-\sigma\left(V_{1,0} \hat{x}_{t}\right) \\
\check{\sigma}_{t}:=\sigma\left(V_{1,0} \hat{x}_{t}\right)-\sigma\left(V_{1,0} x_{t}\right) \\
\hat{\varphi}_{t}:=\varphi\left(V_{2, t} \hat{x}_{t}\right)-\varphi\left(V_{2,0} \hat{x}_{t}\right) \\
\check{\varphi}_{t}:=\varphi\left(V_{2,0} \hat{x}_{t}\right)-\varphi\left(V_{2,0} x_{t}\right) \\
\sigma_{t}:=\sigma\left(V_{1, t} \hat{x}_{t}\right), \varphi_{t}:=\varphi\left(V_{2, t} \hat{x}_{t}\right) \\
\sigma_{t}(0)=0, \varphi_{t}(0)=0
\end{gathered}
$$

In view of the differentiability and the properties $\mathbf{A} \mathbf{3}$ it follows

$$
\begin{gathered}
\hat{\sigma}_{t}=D_{\sigma}\left(V_{1, t} \hat{x}_{t}\right) \tilde{V}_{1, t} \hat{x}_{t}+\eta_{\sigma, t} \\
\hat{\varphi}_{t} \gamma\left(u_{t}\right)=\sum_{i=1}^{q} \gamma_{i}\left(u_{t}\right) D_{i \varphi}\left(V_{2, t} \hat{x}_{t}\right) \tilde{V}_{2, t} \hat{x}_{t}+\eta_{\varphi, t} \gamma\left(u_{t}\right) \\
D_{\sigma}:=D_{\sigma}\left(V_{1, t} \hat{x}_{t}\right):=\left.\frac{\partial}{\partial z} \sigma(z)\right|_{z=V_{1, t} \hat{x}_{t}} \\
D_{i \varphi}:=D_{i \varphi}\left(V_{2, t} \hat{x}_{t}\right):=\left.\frac{\partial}{\partial z} \varphi_{i}(z)\right|_{z=V_{2, t} \hat{x}_{t}} \\
\check{\sigma}_{t}^{\top} \Lambda_{1} \check{\sigma}_{t} \leq \Delta_{t}^{\top} \Lambda_{\sigma} \Delta_{t} \\
\left(\check{\varphi}_{t} \gamma\left(u_{t}\right)\right)^{\top} \Lambda_{2}\left(\check{\varphi}_{t} \gamma\left(u_{t}\right)\right) \leq \lambda_{\max }\left(\Lambda_{2}\right) \check{\varphi}_{t}^{2}\left\|\gamma\left(u_{t}\right)\right\|^{2} \\
\left\|\eta_{\sigma, t}\right\|^{2} \leq l_{1}\left\|\tilde{V}_{1, t} \hat{x}_{t}\right\|^{2},\left\|\eta_{\varphi, t}\right\|^{2} \leq l_{2}\left\|\tilde{V}_{2, t} \hat{x}_{t}\right\|^{2}
\end{gathered}
$$

The weights in (3) are suggested to be adjusted by the following Differential Learning Law:

$$
\begin{aligned}
& \left\{\begin{array}{c}
L_{1, t}^{W}:=2 \frac{d}{d t} \tilde{W}_{1}^{\top} K_{1}^{-1}+ \\
\left(2 \sigma_{t} e_{t}^{\top}\left(C_{\delta}^{+}\right)^{\top} P+\sigma_{t} \sigma_{t}^{\top} \tilde{W}_{1, t}^{\top} P H_{3} P\right)=0 \\
L_{2, t}^{W}:=2 \frac{d}{d t} \tilde{W}_{2}^{\top} K_{2}^{-1}+2 \varphi_{t} \gamma\left(u_{t}\right) e_{t}^{\top}\left(C_{\delta}^{+}\right)^{\top} P+ \\
\varphi_{t} \gamma\left(u_{t}\right) \gamma\left(u_{t}\right)^{\top} \varphi_{t}^{\top} \tilde{W}_{2, t}^{\top} P H_{3} P=0 \\
L_{1, t}^{V}:=2 \frac{d}{d t} \tilde{V}_{1}^{\top} K_{3}^{-1}+2 \hat{x}_{t} e_{t}^{\top}\left(C_{\delta}^{+}\right)^{\top} P W_{1,0} D_{\sigma}+ \\
\hat{x}_{t} \hat{x}_{t}^{T} \tilde{V}_{1, t}^{\top}\left[D_{\sigma}^{\top} W_{1,0}^{\top} P H_{1} P W_{1,0} D_{\sigma}+l_{1} \Lambda_{1}\right]=0 \\
L_{2, t}^{V}:=2 \frac{d}{d t} \tilde{V}_{2}^{\top} K_{4}^{-1}+2 \hat{x}_{t} e_{t}^{\top}\left(C_{\delta}^{+}\right)^{\top} P W_{2,0}\left(D_{t}^{\varphi u}\right)^{\top} \\
+\hat{x}_{t} \hat{x}_{t}^{\top} \tilde{V}_{2, t}^{\top}\left[D_{t}^{\varphi u} W_{2,0}^{\top} P H_{1} P W_{2,0}\left(D_{t}^{\varphi u}\right)^{\top}\right. \\
\left.+l_{2} \gamma\left(u_{t}\right) \gamma\left(u_{t}\right)^{\top} \Lambda_{2}\right]=0 \\
\end{array}\right. \\
& e_{t}:=C \hat{x}_{t}-y_{t} \\
& \tilde{W}_{i, t}:=W_{i, t}-W_{i, 0}, \tilde{V}_{i, t}:=V_{i, t}-V_{i, 0}(i=1,2) \\
& C_{\delta}^{+}:=\left(C^{\top} C+\delta I\right)^{-1} C^{\top}, N_{\delta}:=\left(C^{\top} C+\delta I\right)^{-1}, \delta>0 \\
& H_{1}:=\left(\delta N_{\delta} \Lambda_{1}^{-1} N_{\delta}^{\top}+C_{\delta}^{+} \Lambda_{y}^{-1}\left(C_{\delta}^{+}\right)^{\top}\right) \\
& H_{3}:=\left(\delta N_{\delta} \Lambda_{3}^{-1} N_{\delta}^{\top}+C_{\delta}^{+} \Lambda_{y}^{-1}\left(C_{\delta}^{+}\right)^{\top}\right) \\
& D_{t}^{\varphi u}:=\sum_{i=1}^{q} \gamma_{i}\left(u_{t}\right) D_{i \varphi}\left(V_{2, t} \hat{x}_{t}\right)
\end{aligned}
$$

with any nonzero initial weights $V_{1,0}$ and $V_{2,0}$. Define the state estimation error by $\Delta_{t}:=\hat{x}_{t}-x$ and

$$
\begin{gathered}
\rho^{+}:=\limsup _{t \rightarrow \vec{s}^{\infty}} E\left\{\rho_{t}\right\} \\
\rho_{t}:=4\left(\bar{W}_{t}^{y}\right)^{\top} \Lambda_{y} \bar{W}_{t}^{y}+\operatorname{tr}\left\{\sigma^{x} \sigma^{x} P\right\}+ \\
2 \lambda_{\max }\left(\Lambda_{2}\right) \tilde{\varphi}^{2}\left(\gamma_{0}^{2}+\gamma_{1}^{2}\left\|u_{t}\right\|^{2}\right)+ \\
\left\|\Lambda_{f}\right\|\left(f_{0}+f_{1}\left\|x_{t}\right\|^{2}+f_{2}\left\|u_{t}\right\|^{2}\right)
\end{gathered}
$$

Theorem 1 (Averaged Upper Bound) If, under the assumptions $\boldsymbol{A 1 - A 7 , ~ t h e ~ l e a r n i n g ~ l a w ~ ( 7 ) ~ i s ~ a p p l i e d , ~}$ 
then the following propertie of the corresponding state estimation process is guaranteed: the averaged quadratic state estimation error turns out to be bounded with the probability one:

$$
\limsup _{t \rightarrow \infty} t^{-1} \int_{\tau=0}^{t} \Delta_{\tau}^{\top} Q_{0} \Delta_{\tau} d \tau \stackrel{a . s .}{\leq} \rho^{+}
$$

\section{Theorem 2 (On Expected Quadratic Variation)}

The assumptions A1-A'7 imply

$$
\begin{gathered}
{\left[1-\mu / \sqrt{E\left\{\Delta_{t}^{\top} P \Delta_{t}\right\}}\right]_{+t \rightarrow \infty} 0} \\
\mu:=\sqrt{\rho^{+} / \lambda_{\min }\left(P^{-1 / 2} Q_{0} P^{-1 / 2}\right)}
\end{gathered}
$$

where the operator $[\cdot]_{+}$is defined as $[z]_{+}:=$ $\left\{\begin{array}{ll}z & \text { if } \quad z>0 \\ 0 & \text { if } \quad z \leq 0\end{array}\right.$.

\section{Simulation}

\subsection{Example 1 (benchmark nonlinear system)}

The nonlinear stochastic process generated by

$$
\left\{\begin{array}{l}
d x_{1, t}=\left[-5 x_{1, t}+3\left|x_{2, t}\right|+u_{1, t}\right] d t+0.088 d \bar{W}_{1, t} \\
d x_{2, t}=\left[-10 x_{2, t}+2\left|x_{1, t}\right|+u_{2, t}\right] d t+0.088 d \bar{W}_{2, t} \\
d y_{t}=C d x_{t}+0.0088 d \bar{W}_{t} \\
x_{1,0}=0.1, x_{2,0}=-0.2
\end{array}\right.
$$

is considered. The programmed controls are the sinewave and the saw-tooth periodic functions, that is, $u_{1 . t}=u_{1,0} \sin (\omega t), u_{1,0}=\omega=1$ and $u_{2, t}=u_{2,0}$. $(t-k \tau), t \in[k \tau,(k+1) \tau), u_{2,0}=\tau=0.25, \quad k=$ $0,1,2, \ldots$ The DNN applied for the identifications is as follows

$$
\begin{gathered}
d \hat{x}_{t}=\left[A \hat{x}_{t}+W_{1, t} \sigma\left(V_{1, t} \hat{x}_{t}\right)+W_{2, t} \varphi\left(V_{2, t} \hat{x}_{t}\right) u_{t}\right. \\
\left.+K\left(y_{t}-C \hat{x}_{t}\right)\right] d t \\
\hat{x}_{1,0}=0.2, \hat{x}_{2,0}=0.1 \\
\sigma_{i}(z)=2 /\left(1+\exp \left(-r_{s} z_{i}\right)\right)-1, r_{s}=0.8, r_{f}=0.5 \\
\varphi_{1,2}=\varphi_{2,1}=0 \\
\varphi_{i, i}(z)=0.5 /\left(1+\exp \left(-r_{f} z_{i}\right)\right)-0.05
\end{gathered}
$$

and the weights $W_{1, t} \in \mathbb{R}^{2 \times 4}, V_{1, t} \in \mathbb{R}^{4 \times 2}, W_{2, t}, V_{2, t} \in$ $\mathbb{R}^{2 \times 2}$ are adjusted by the learning law (7) with $q=$ $2, \gamma_{i}(u)=u_{i}(i=1,2)$. The selected parameter values are

$$
\begin{gathered}
A=\left(\begin{array}{cc}
-3.9 & 0 \\
0 & -2
\end{array}\right), K=\left(\begin{array}{l}
5 \\
5
\end{array}\right), l_{1}=l_{2}=0.3 \\
\Lambda_{1}=\Lambda_{2}=\Lambda_{3}=\Lambda_{y}=0.3 I, \quad k_{1, v_{1}}=k_{1, v_{2}}=20000 \\
k_{1, w_{1}}=1500, k_{1, w_{2}}=800 \\
R=0.0122\left(\begin{array}{cc}
1 & 0 \\
0 & 1
\end{array}\right), Q=\left(\begin{array}{cc}
0.30643 & 0.12043 \\
0.12043 & 4.80043
\end{array}\right) \\
P=\left(\begin{array}{cc}
0.05528 & 0.00362 \\
0.00362 & 0.05530
\end{array}\right)
\end{gathered}
$$

and the initial weight values equal to

$$
\begin{gathered}
W_{1,0}=\left(\begin{array}{cccc}
0.5 & 0.1 & 0 & 2 \\
0.2 & 0.5 & 0.6 & 0
\end{array}\right), V_{1,0}=\left(\begin{array}{cc}
1 & 0.5 \\
0.3 & 1.3 \\
0.5 & 1 \\
0.2 & 0.6
\end{array}\right) \\
W_{2,0}=\left(\begin{array}{cc}
0.5 & 0.1 \\
0 & 0.2
\end{array}\right), V_{2,0}=\left(\begin{array}{cc}
1 & 0.1 \\
0.1 & 2
\end{array}\right)
\end{gathered}
$$

The correspondent simulation results, realized only with the use of 6 neurons, are depicted at the figures Fig.1-Fig.4. The value of the performance index $I_{t}$, obtained after $t=20 \mathrm{sec}$., is equal to

$$
I_{t=20}=t^{-1} \int_{\tau=0}^{t}\left\|\Delta_{\tau}\right\|^{2} d \tau=0.00137
$$

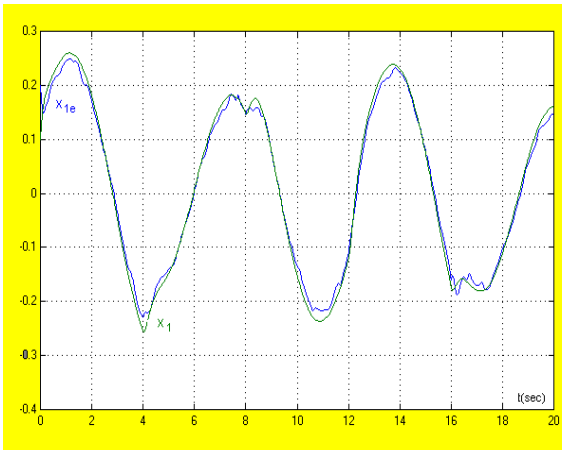

Figure 1: X1 and X1e.

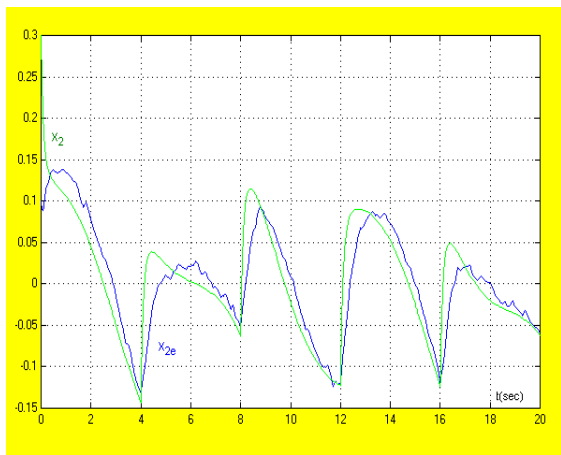

Figure 2: X2 and X2e.

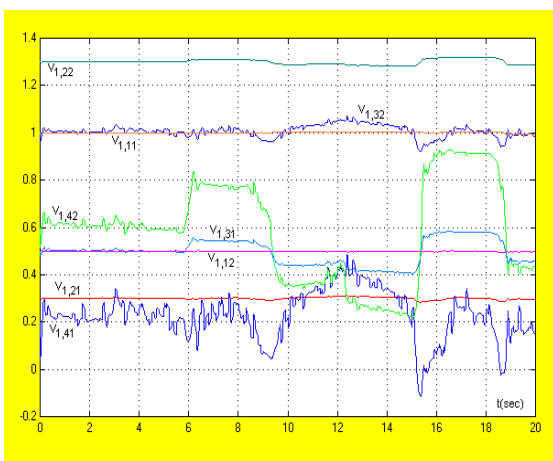

Figure 3:V1 Weights. 


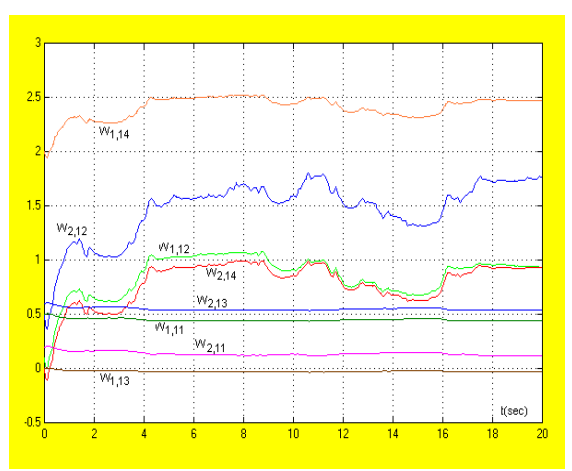

Figure 4:W1 Weights.

\subsection{Example 2 (a double tank liquid system)}

The model, considered below, describes a double tank liquid system and corresponds to a standard laboratory process including two water tanks in series (see Fig.5)

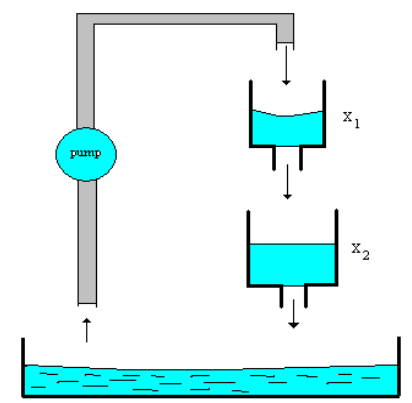

Figure 5: The double tank processes.

The input signal $u_{t}$ is the voltage to the pump and the output signal $y_{t}$ is the level of the lower tank. The control goal is to control the level $x_{2}$ of the lower tank and indirectly the level $x_{1}$ of the upper tank. In the general case, these two tanks levels are both measurable. But here we will consider the situation when the only level $x_{2}$ in the lower tank can be estimated directly. Our aim is to estimate the level $x_{1}$ of the upper tank based only on the output measurements corrupted by " a white noise". The following state space description holds:

$$
\left\{\begin{array}{l}
d x_{1} / d t=-\alpha_{1} \sqrt{x_{1}}+\beta u_{t} \\
d x_{2} / d t=\alpha_{1} \sqrt{x_{1}}-\alpha_{2} \sqrt{x_{2}} \\
y=x_{2}+0.01 W_{y}
\end{array}\right.
$$

where $u_{t}$ is the control variable (water flow) selected as " a single step-function", $x=\left(x_{1}, x_{2}\right)^{\top}$ are the states of this process, $W_{y}$ is the white noise component, $y$ is the measurable output. The parameters of the process are as follows: $\alpha_{1}=1.5, \alpha_{2}=1.5, \beta=0.3$. And the parameters of the DNN observer are as follows:

$$
\begin{gathered}
\sigma_{i}(z)=2 /\left(1+\exp \left(-r_{s} z_{i}\right)\right)-1, r_{s}=8, r_{f}=8 \\
\varphi_{1,2}=\varphi_{2,1}=0 \\
\varphi_{i, i}(z)=0.5 /\left(1+\exp \left(-r_{f} z_{i}\right)\right)-0.05
\end{gathered}
$$

and the weights $W_{1, t} \in \mathbb{R}^{2 \times 4}, V_{1, t} \in \mathbb{R}^{4 \times 2}, W_{2, t}, V_{2, t} \in$ $\mathbb{R}^{2 \times 2}$ are adjusted by the learning law (7) with $q=$ $2, \gamma_{i}(u)=u_{i}(i=1,2)$. The selected parameter values are

$$
\begin{aligned}
& A=\left(\begin{array}{cc}
-5.89 & 0.4 \\
0 & -2
\end{array}\right), K=5\left(\begin{array}{l}
1 \\
1
\end{array}\right) \\
& \Lambda_{1}=\Lambda_{2}=\Lambda_{3}=\Lambda_{y}=0.3 I, l_{1}=l_{2}=0.3 \\
& k_{1, w_{1}}=15000, k_{1, w_{2}}=8000 \\
& k_{1, v_{1}}=20000, k_{1, v_{2}}=200000 \\
& R=0.0122\left(\begin{array}{ll}
1 & 0 \\
0 & 1
\end{array}\right), Q=\left(\begin{array}{ll}
0.30643 & 0.12043 \\
0.12043 & 4.80043
\end{array}\right) \\
& P=\left(\begin{array}{ll}
0.00256 & 0.00026 \\
0.00026 & 0.01108
\end{array}\right)
\end{aligned}
$$

We obtained the simulation results depicted at Figures 6-7. The value of the performance index $I_{t}$, obtained after $t=20 \mathrm{sec}$.,is equal to

$$
I_{t=20}=t^{-1} \int_{\tau=0}^{t}\left\|\Delta_{\tau}\right\|^{2} d \tau=0.00007717
$$

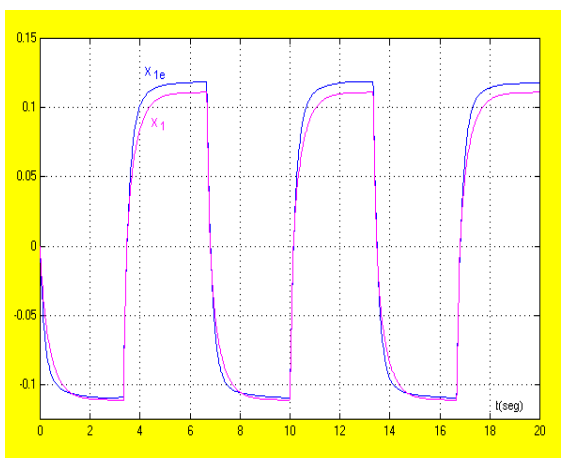

Figure 6: X1 and X1e

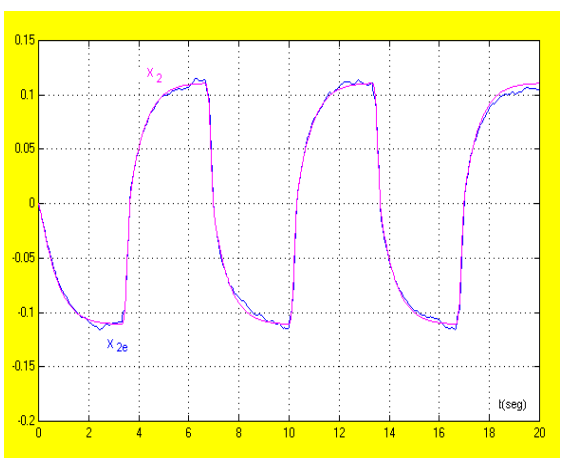

Figure 7: X2 and X2e

\section{Conclusion}

In this paper a new asymptotic differential neuro observer is proposed to estimate the states of continuous time stochastic process without a priory knowledge of the plant-structure. The corresponding weight matrices of this DNN - observer are adjusted by a special differential learning law containing two terms: the first one 
is standard resemble to the "back-propagation" scheme and the second one is proportional to the output error. Even the sigmoidal nonlinearities are bounded and, in the first glance, the hidden layer weights variation may not effect the identification quality, the presented theoretical study (as well as the corresponding simulations) show that the hidden layer weights updating significantly improves the observation process. Stochastic Lyapunov analysis (with the use of the Itô's formula) is applied to prove the existence of the upper bound to the averaged quadratic estimation error. The boundness "in average" is stated also for the weight matrices which are time varying during all learning time in the opposite to the static DNN which weights obligatory converge. The simulation results also demonstrate the high effectiveness of the suggested DNN-observer application even its structure is simple enough( containing the only 4 neurons). The next natural study step is to extend this approach to the case of the Adaptive Tracking based on such DNN-observers, that is, to consider the workability of this DNN in a stochastic close-loop system.

\section{References}

[1] Agarwal M., 1997, A Systematic Classification of Neural Network Based Control, IEEE Control Systems Magazine, 17, 75-93.

[2] Gard T.C., Introduction to Stochastic Differential equations, 1988, Marcel Dekker Inc., NY and Basel.

[3] Duncan T.E., Lei Guo and B. Passik-Duncan, 1999, Adaptive Continuous-time Linear Quadratic Gaussian Control, IEEE Transaction on $A C, 44$ (9), 1653-1662.

[4] Elenayar and Shin , 1994, Approximation and Estimation of Nonlinear Stochastic Dynamic Systems Using Radial Basis Function Neural Network, IEEE Trans. on Neural Networks, 5, 594-603.

[5] Hopfield J.J., 1984, Neurons with Grade Responce Have Collective Computational Properties Like Those of a Two-state Neurons, Proc. Nat. Academy Sci., USA, 8, 3088-3092.

[6] Hunt K.J., Sharbaro D., Zbikovski R. and P.J.Gawthrop, 1992, Neural Networks for Control Systems - A Survey", Automatica, 28, 1083-1112.

[7] Haykin S., 1994, Neural Networks: A Comprehensive Foundation, IEEE Press, NY.

[8] Isidori A., Nonlinear Control Systems, 3.rd Ed., 1995, New York, Springer- Verlag.

[9] Kosmatopulos E.B. and M.Christodolou, 1994, The Boltzmann g-RHONN: A Learning Machine for estimating Unknown Probability Distributions, Neural Networks, 7 (2), 271-278.
[10] Krstić M., Kanellakopulos I. and P.Kokotovich, Nonlinear and Adaptive Control Design, 1995, John Walley \& Sons, NY.

[11] Lewis F.L. and T.Parisini, 1998, Neural Network Feedback Control with Guaranteed Stability, Int. J. Control, 70 , 337-340.

[12] Ljung L. System for Identification. Theory for the Users (Second Edition). 1999. Prentice Hall PTR, Upper Saddle River, NJ 07458.

[13] Ljung L. and S.Gunnarson, Adaptation and Tracking in System Identification - A Survey, 1990, Automatica, $\mathbf{2 6}$, 7-21.

[14] Narendra K.S. and S.M.Li, 1998, Control of Nonlinear Time-Varying Systems Using NN, Proc. of 10-th Yale Workshop on Adaptive and learning Systems, 218.

[15] Parisini T. and R.Zoppoli, 1994, Neural Networks for Feedback Feedforward Nonlinear Control Systems, IEEE on $N N, 5,436-449$.

[16] Poznyak A.S. and Wen Yu, 2000, "Robust Asymptotic Neuro-Observer with Time Delay Term", Int.Journal of Robust and Nonlinear Control, 10, 535559 .

[17] Poznyak A.S, E.N. Sanchez, Wen Yu and J.P. Perez, 1999, Nonlinear Adaptive Trajectory Tracking Using Dynamic Neural Networks, IEEE on NN, 10 (6), 1402-1411.

[18] Poznyak A.S., Martinez-Guerra R. and A.OsorioCordero, 2000, Robust High-gain Observer for Nonlinear Closed-Loop Stochastic Systems, Mathematical Methods in Engineering Practice, 6, pp.31-60.

[19] Poznyak A.S., 2000, A New Version of the Strong Law of Large Number for Dependent Vector Processes with Decreasing Correlation, Proceedings of CDC-2000, Sydney, 2881-2882.

[20] Poznyak A. and L.Ljung, 2001, On-line Identification and Adaptive Trajectory Tracking for Nonlinear Stochastic Continuous Time Systems Using Differential Neural Networks, Automatica IFAC, especial issue (submitted).

[21] Rovithakis G.A. and M.A.Cristodoulou, 1994, Direct Adaptive regulation of Unknown Plants using DNN, IEEE on SMC, 24 (3), 400-412.

[22] Rovithakis G.A. and M.A.Cristodoulou, 2000, Adaptive control with Recurrent High-Order Neural Networks: Theory and Applications, Springer.

[23] Sandberg I.W., 1991, Approximation Theorems for Discrete Time Systems, IEEE on AC, 38, 564-566. 


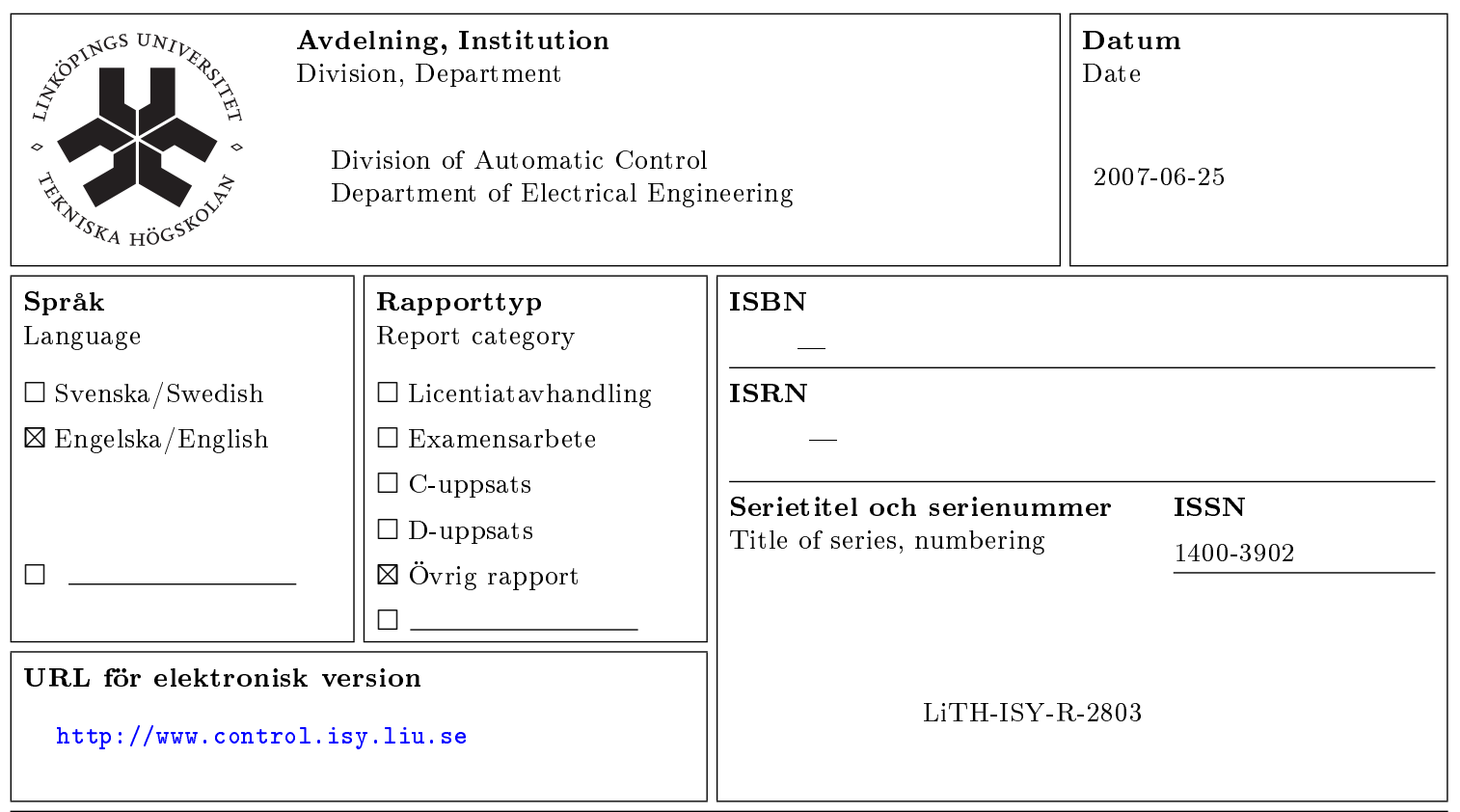

Titel Robust high-gain DNN observer for nonlinear stochastic continuous time systems

Title

(1)

Författare D.A. Murano, A.S. Poznyak, Lennart Ljung

Author

\section{Sammanfattning}

Abstract

A class of nonlinear stochastic processes satysfying a "Lipschitz-type strip condition" and supplied by a linear output equation, is considered. Robust asymptotic (high-gain) state estimation for nonlinear stochastic processes via differential neural networks is discussed. A new type learning law for the weight dynamics is suggested. By a stochastic Lyapunov-like analysis (with Ito formula implementation), the stability conditions for the state estimation error as well as for the neural nertwork weights are established. The upper bound for this error is derived. The numerical example, dealing with "module"-type nonlinearities, illustrates the effectiveness of the suggested approach. 